\title{
A BARRELLED SPACE WITHOUT A BASIS
}

\author{
N. J. KALTON
}

Abstract. An example is given of a separable, barrelled, nuclear, bornological Ptak space which has no Schauder basis.

A sequence $\left\{x_{n}\right\}$ in a locally convex space $E$ is a basis if each $x$ in $E$ can be expressed uniquely in the form $x=\sum_{i=1}^{\infty} a_{i} x_{i}$; Singer [5] has given an example of a separable locally convex space which does not possess a basis. In this note I shall give another example which has the additional properties of being barrelled, bornological, nuclear and a Ptak space; this also partially answers a question raised in [2].

It seems desirable to introduce another form of separability in locally convex spaces: $E$ will be called $\omega$-separable if it possesses a subspace $G$ of countable dimension and such that every member of $E$ is the limit of a sequence in $G$. Thus if $E$ has a basis, $E$ is $\omega$-separable, and if $E$ is $\omega$-separable then $E$ is separable. Let $\boldsymbol{N}$ denote the cardinal of the continuum and let $\operatorname{card}(X)$ denote the cardinal of any set $X$. If $E$ is $\omega$-separable then $\operatorname{card}(E)$ is less than the cardinal of the set of all sequences in $G$; as $\operatorname{card}(G)=\boldsymbol{N}$, $\operatorname{card}(E)=\boldsymbol{N}$.

Let $K$ be the field of real numbers or of complex numbers.

Theorem. $K^{\aleph}$ is barrelled, bornological, nuclear and a Ptak space; it is separable but not $\omega$-separable, and so does not possess a basis.

Proof. $K^{N}$ has a weak topology and is complete; hence $K^{N}$ is a Ptak space (see $[4$, p. 162]). By the Mackey-Ulam theorem ([3, $\$ 28.8]$ ) it is bornological as $\boldsymbol{N}$ is not strongly inaccessible; a complete bornological space is barrelled. The product of nuclear spaces is nuclear $\left[4\right.$, p. 102] and so $K^{\aleph}$ is nuclear. Finally, $K^{\aleph}$ is separable by Theorem 7.2, p. 175 of [1] but card $\left(K^{\aleph}\right)=2^{\aleph}>\aleph$, and so $K^{\aleph}$ cannot be $\omega$-separable.

The question which naturally arises is: does every $\omega$-separable locally convex space possess a basis? Singer's example, the weak ${ }^{*}$-dual of the Banach space $m$ of all bounded sequences, is not $\omega$-separable; ${ }^{1}$ this follows from the results of [5] or from the fact that $m^{*}$ contains a copy of the Stone-Cech compactification of the integers, and so has cardinality $2^{\aleph}$.

Received by the editors February 6, 1970.

AMS 1969 subject classifications. Primary 4601.

Key words and phrases. Locally convex spaces, basis, separable, barrelled, nuclear.

"I am grateful to the referee for pointing this out. 


\section{REFERENCES}

1. J. Dugundji, Topology, Allyn and Bacon, Boston, Mass., 1966. MR 33 \#1824

2. N. Kalton, Schauder decompositions and completeness, Bull. London Math. Soc. 2 (1970), 34-36.

3. G. Köthe, Topologische Lineare Räume. I, Die Grundlehren der math. Wissenschaften, Band 107, Springer-Verlag, Berlin, 1960. MR 24 \#A411.

4. H. H. Schaefer, Topological vector spaces, Macmillan, New York, 1966. MR 33 \#1689.

5. I. Singer, On the basis problem in linear topological linear spaces, Rev. Roumaine Math. Pures Appl. 10 (1965), 453-457. MR 33 \#3081.

Lehigh University, Bethlehem, Pennsylvania 18015 